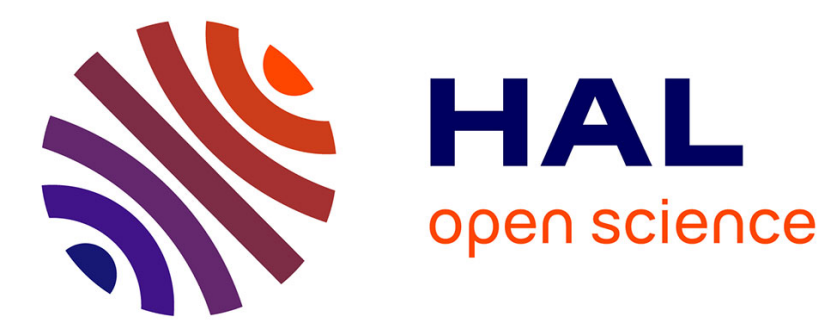

\title{
Population characteristics and the decision to convert to organic farming
}

\author{
Q. Xu, S. Huet, Eric Perret, I. Boisdon, G. Deffuant
}

\section{To cite this version:}

Q. Xu, S. Huet, Eric Perret, I. Boisdon, G. Deffuant. Population characteristics and the decision to convert to organic farming. Social simulation conference, Aug 2018, Stockholm, Sweden. 16 p. hal-01901792

\section{HAL Id: hal-01901792 \\ https://hal.science/hal-01901792}

Submitted on 23 Oct 2018

HAL is a multi-disciplinary open access archive for the deposit and dissemination of scientific research documents, whether they are published or not. The documents may come from teaching and research institutions in France or abroad, or from public or private research centers.
L'archive ouverte pluridisciplinaire HAL, est destinée au dépôt et à la diffusion de documents scientifiques de niveau recherche, publiés ou non, émanant des établissements d'enseignement et de recherche français ou étrangers, des laboratoires publics ou privés. 


\title{
Population characteristics and the decision to convert to organic farming
}

\author{
Xu Q. ${ }^{[1]}$, Huet S. ${ }^{[2]}$, Perret E. ${ }^{[1]}$, Boisdon I. ${ }^{[1]}$, Deffuant G. ${ }^{[2]}$ \\ ${ }^{1}$ UCA, AgroParisTech, Inra, Irstea, VetAgro Sup, Territoires; ${ }^{2}$ Lisc - Irstea \\ 9 avenue Blaise Pascal, 63178 Aubière, France \\ qing . xu@irstea.fr
}

\begin{abstract}
We revisit some ideas of why farmers do not convert to organic farming from our previous article with a dynamic individual based model. In this model, an agent's decision on transitioning to organic is based on the comparison between the satisfaction with its current situation and the potential satisfaction with an alternative farming strategy. A farmer agent's satisfaction is modeled with the Theory of Reasoned Action. It is computed by comparing the agent's outcomes over time and comparing its current outcome against those of other agents to whom it lends great credibility ('important others'). The first study is based on prototypical farm populations. In this paper, the predicted conversion rate is studied with some French "cantons" having different practice intensities. The model is initialized with dairy farmers' data in these "cantons" in 2000. The results show that the "cantons" characteristics have great impact on the virtual adoption rate. Intensive "cantons" convert less than extensive ones. Extensive farms having not very good environmental outcomes seem to convert the most.
\end{abstract}

Keywords: organic farming, decision making, major change, Theory of Reasoned Action, agent-based model, social influence, credibility.

\section{Introduction}

The recent dairy crisis combined with increasing consumer demand for organic food have made conversion to organic farming socially and economically interesting for dairy farmers [1], [2]. However, many farmers still do not convert. Why?

Recently, the conversion to organic farming has been qualified as a major change [3] or a transformational adaptation [4], [5], as well as a social movement [3], [6]-[9]. Conversion often implies strong changes in a farmer agent's worldview and social network, and generally begins with a strong need for change [3], [10]. Such a change that engages a number of social processes involving the agent, its peers and its environment has been rarely studied [11].

Agent-based modeling [12] or individual-based modeling [13] appear to be relevant and well-geared to help identifying the main drivers that can explain the observed dynamics. However, as pointed out in [14], [15], none of current agent models is well fit to represent the decision process about a major change that is at stake in the 
conversion to organic farming. This is the reason why [15] has proposed a dynamic version of the Theory of Reasoned Action (TRA); and showed its potential to explain why farmers do not convert to organic farming in a prototypical farmer population. This paper aims at evaluating this model when running on more realistic farmer populations designed from the French Agricultural Census data (RGA) 2000. The purpose is mainly to study how the conversion rate evolves differently in various farmer populations. The main original features of the model are the following:

- Like [16], we propose a dynamic model of TRA [17] to compute an agent's satisfaction. In the model, a decision on conversion is based on comparing the agent's satisfaction with its current strategy against its potential satisfaction with an alternative one. These satisfactions are computed with the attitude and the subjective norm related to the current or alternative strategy. Both attitude and subjective norm are dynamic and based on the difference in practice outcomes. Consistently with TRA [17], our model integrates decomposed variables: attitude and subjective norm - instead of global variables as in [16]. A farmer agent's evaluation is thus based on its concrete strategy and practice instead of an abstract general opinion. The evaluation relates to its own experiences and its peers' strategies and practices.

- An agent's attitude about its current strategy is modelled as the difference between its past outcomes with current strategy and its current outcome. This choice is grounded in [18], [19] who argue that a farmer agent's earlier practices are very influential for its decision on a future practice. Regarding an alternative strategy which can be new to the agent, the attitude is the difference between its past outcomes with current strategy and the outcomes of other peers with a similar farm and adopting the alternative or the outcome of the alternative in the media.

- The subjective norm involves a comparison with peers, weighted by their credibility. This credibility is based on the difference between outcomes (i.e. total production).

- As [20] showed that farmers co-construct their practices. An agent updates its practice by copying the performances of its credible peers with a similar farm.

- Finally, an important feature of our model is tied to the decision process about a "major change". It is assumed that a major change is only considered in critical situations where an agent faces high costs (economic, cognitive, emotional, etc.). Otherwise, agents do not even consider changing their major options. In a stable period, if a farmer agent is satisfied enough with its current farming strategy, it does not envisage an alternative one. Only a certain level of frustration or critical event (succession, major change in the farm, etc.) will evoke a consideration of an alternative to a farmer's current strategy.

In [15], the model is studied with prototypical farm populations and shows different reasons for the lack of conversion to organic farming. In this work, the model is explored further by an initialization with six French "cantons" (about 100 farmers) varying in their dairy farm type distributions. The adoption degree is compared among these populations to clarify the effect of population features. In general, intensive "cantons" convert less than extensive ones. Particularly, extensive farms having not very good environmental outcomes seem to convert the most. 
After presenting the model's principles with partially ODD (Overview, Design concepts, Details) protocol [21], we outline the model's behaviors and some explanations before going on to synthesis, and discuss our conclusions.

\section{Materials and Methods}

\subsection{The model}

\section{Basic elements.}

Farmer.

The model studies the evolution of a population with $N$ farmer agents. Each agent is characterized by its farm; its farming strategy; its practice defined on several dimensions $i$ which are evaluated through performances; the importance $W_{i}$ given to each dimension of practice; the credibility $(C(f, v))$ it lends to each other agent; its memory of applied strategies and practices during the last $M$ periods; its satisfactions with current farming strategy $\left(I_{C}\right)$ and with an alternative one $\left(I_{A}\right)$; and its duration for staying with a strategy $(D C)$ and for being dissatisfied for a strategy after being stable $(D D)$.

$D C$ and $\boldsymbol{D D}$ capture the duration between two events related to the decision process. $D D$ counts an agent's dissatisfaction duration with its current strategy. In the model, an agent has to be dissatisfied long enough with its current strategy to change it. $D C$ counts the duration since last strategy change. An agent cannot consider changing strategy again even if the agent is dissatisfied with it during the confirmation period. This is consistent with the theory of innovation diffusion [22] in which an agent has a confirmation period just after adopting a new strategy. Both counters are necessary to simulate an agent's stability and consistency. The corresponding delayed action of both counters can only occur when the counter is above the parameter $T D$.

Except for $W_{i}$, all these attributes of a farmer agent are dynamic during the simulation, and are described in detail below.

Credibility. Each agent $f$ gives a credibility $C(f, v)$ to another agent $v$ by comparing their outcomes. Credibility is between 0 (not credible at all) and 1 (very credible).

Satisfaction. Each agent has a satisfaction with its current farming strategy $\left(I_{C}\right)$ that corresponds to an evaluation of that strategy. It may also evaluate an alternative strategy in certain cases and have a satisfaction for it $\left(I_{A}\right)$. Satisfaction with a farming strategy lies between 0 (not satisfied at all) and 1 (very satisfied).

If an agent is satisfied with its current farming strategy, it does not consider an alternative. Otherwise, its satisfactions with its current farming strategy $\left(I_{C}\right)$ and with an alternative one $\left(I_{A}\right)$ are computed and compared. If $I_{A}$ is higher enough than $I_{C}$, the agent will change its farming strategy. $I_{C}$ is thus computed at every iteration, whereas $I_{A}$ is only computed when a stable agent is dissatisfied with its current farming strategy. 
In accordance with TRA, the satisfaction $I_{S}$ with a farming strategy $S$ depends on two elements: attitude $A_{S}$ and subjective norm $S N_{S}$ toward $S$. In the original theory, the interaction between these two elements varies with different agents facing different situations. In order to keep the model simple, satisfaction is assumed as the average value of these two elements.

$$
I_{S}=\frac{A_{S}+S N_{S}}{2}
$$

Both attitude and subjective norm lie between -1 (very negative attitude/subjective norm concerning the farming strategy to evaluate) and 1 (very positive attitude/ subjective norm concerning the farming strategy to evaluate). They are computed with farms' outcomes, farmers' strategies, and credibility. See the section 'Farmers' dynamics" for the computation details.

Considering the value range of attitude and subjective norm towards a farming strategy, the satisfaction should also lie between -1 and 1 . However, to facilitate other calculations, the satisfaction is normalized between 0 and 1 .

Practice. As stated above, the term "practice" in the model is not really an agent's actual practice, but the way the agent evaluates it, i.e. its performance. A practice is evaluated over two dimensions: the level of output production (i.e. the productivity impact, in our case milk production), and the level of environmental amenities production (i.e. the environmental impact), respectively called productivity performance $\left(P_{0}\right)$ and environmental performance $\left(P_{l}\right)$ in the paper. Both $P_{0}$ and $P_{l}$ lie between 0 (very bad on this practice dimension) and 1 (very bad on this practice dimension).

Importance given to each dimension. The importance given to productivity dimension is termed $W_{0}$, and the one given to environmental dimension is termed $W_{l}$. $W_{0}$ and $W_{l}$ lie between 0 (not important at all) and 1 (most important). They sum to 1 .

$$
W_{0}+W_{l}=1
$$

Importance defines an agent's personal values. An agent uses its own lens to judge the information it receives and the other agents it meets. In this model, both $W_{0}$ and $W_{l}$ are kept constant if an agent does not change its farming strategy.

Farming strategy. It is defined by the importance that a farmer gives to each dimension of practice. Two farming strategies are considered: organic and conventional. The organic strategy means lending more importance to environmental dimension and less to productivity dimension, whereas the conventional strategy does the contrary. It is assumed that when a farmer agent changes its strategy, it changes accordingly the importance given to each dimension.

Farm.

A farm has three attributes: its farming total production (productivity outcome) $T_{0}$, its environmental amenities outcome $T_{l}$ and its reference $R . R$ is the maximum possible productivity performance considering a farm's all characteristics and evolution. Interviews and experts' arguments show that conventional farms' references are grounded on the negotiations with dairy enterprises (often expressed by "quota" in Europe in the past). Organic farms have more constraints in terms of reference due to stricter 
regulations. So a conventional farm $f$ 's reference is considered as its farmer $f$ 's initial productivity performance $P_{0}^{f}(t=0)$ and that for an organic farm is a function $l$ of $P_{0}^{f}$ $(t=0)$.

$$
R^{f}=\left\{\begin{array}{c}
P_{0}^{f}(\mathrm{t}=0) \text { if } f \text { is a conventional farm/farmer } \\
l\left(P_{0}^{f}(\mathrm{t}=0)\right) \text { otherwise }
\end{array}\right.
$$

The implementation of a farm may need more attributes for different use cases. The detailed computation of $T_{0}$ and $T_{1}$ are defined in the model's implementation (see 2.2).

Media.

When an agent is dissatisfied with its current farming strategy and looks for an alternative, it first searches in the population for other agents having similar characteristics but applying an alternative strategy. If it cannot find one, it has access to the media for an stereotype alternative model which depends on the farm's current outcomes.

\section{Dynamics.}

Overview of a farmer's dynamics over years.

One time-step (iteration) $t->t+1$ represents one year, i.e. farmers decide their farming strategies, their practices, and so on, once a year. During an iteration, farmers' update order is picked up at random by a uniform law.

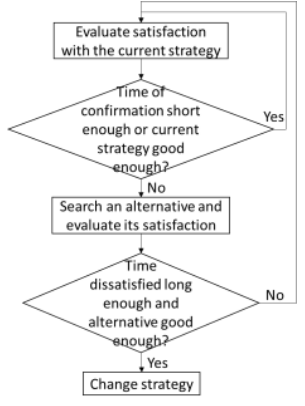

Fig. 1. - Overview of the farmer's update

For each iteration \{

Generate the order of the population

For each agent $f$ in the population \{

Compute $I_{C}$

If $D C>T D$ and $I_{C}<T A$, compute $I_{A}$

If $D D>T D$ and $I_{A}>I_{C}+T O$, change strategy and update $W_{0}, W_{l}$

For each agent $v$ that is different from agent $f$ in the population, compute $C(f, v)$

Compute $\left.P_{0}, P_{1} \quad\right\}$ \}

Algorithm 1- Population updating loop. $I_{C}$ is the satisfaction with a current strategy; $I_{A}$ is the satisfaction with an alternative one. $D C$ is an agent's confirmation duration; $D D$ is an agent's dissatisfaction duration. $T D$ is the minimum time of dissatisfaction 
before considering the alternative. $T A$ is threshold of $I_{C}$ to consider an alternative. TO is the threshold of $I_{A}$ to change strategy. $W_{0}$ is the importance given to productivity performance, $W_{l}$ is the importance given to environmental performance. $C(f, v)$ is the credibility that agent $f$ gives to agent $v . P_{0}$ is the productivity performance, $P_{l}$ is the environmental performance.

As shown in Figure 1 and the pseudocode of algorithm 1, during each iteration, an agent evaluates its satisfaction with its current farming strategy. If the agent is in a stable period and is satisfied with its current strategy, it does not consider a change. Otherwise, the agent looks for an alternative and evaluates it. If the agent has been dissatisfied for long enough and the alternative is good enough, it will change. Otherwise, the agent stays with its current farming strategy. It will then update its credibility given to other agents and its practice. See the detail in the following.

\section{Credibility update.}

Every two agents' relationship is characterized by the credibility one gives to another, and depends on an agent's personal view of its difference to another in outcome (i.e. total production). For agent $f$, its difference to agent $v$ is the sum of difference on each outcome dimension weighted by the importance given to that dimension.

$$
D_{v}^{f}=\sum_{i=1}^{2}\left(W_{i}^{f}\left(T_{i}^{v}-T_{i}^{f}\right)\right)
$$

The credibility that agent $f$ gives to agent $v$ is calculated with $f$ 's difference to $v$ :

$$
C_{v}^{f}=\frac{1}{1+e^{-\alpha D_{v}^{f}}}
$$

with $\alpha$ the parameter to characterize the slope of logistic function.

In Figure 2, agent $f$ 's difference and credibility to $v$ are respectively plotted on the $\mathrm{x}$-axis and $\mathrm{y}$-axis. When the difference is negative, it means that $v$ has a worse outcome than $f$, thus $f$ gives little credibility to $v$. When the difference is positive, $v$ has a better outcome than $f$, thus $f$ gives big credibility to $v$.

The lines with different colors represent $\alpha$ variations to characterize the bias degree that an agent has for others with better outcomes. When $\alpha$ is small, the bias is small. An agent tends to give same credibility to others, whether or not with better outcomes. If $\alpha$ is big, the bias is strong. Only others with better outcomes are credible.

In the model, every two agents are connected. The credibility depends on an agent's perceived difference in outcome to another and it is then used to update the agent's outcome which can change the perceived difference. Thus, these elements are dynamic.

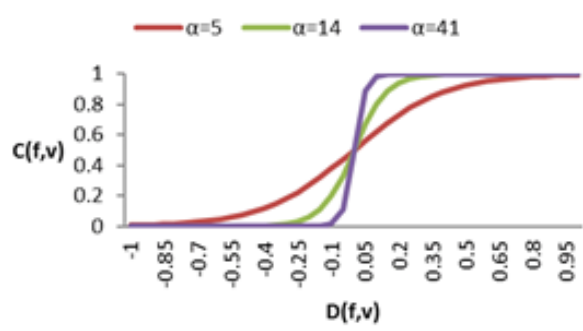


Fig. 2. - The credibility ( $y$-axis) agent $f$ gives to $\mathrm{v}$ depends on $f$ 's difference ( $x$-axis) to $v$ for three values of parameter $\alpha$ (different-colored lines)

Farming strategy change.

An agent changes its farming strategy according to its satisfaction evaluations with its current strategy $\left(I_{C}\right)$ and with the alternative one $\left(I_{A}\right)$. If an agent is in a stable state (its confirmation duration $D C>$ threshold $T D$ ) and it is still dissatisfied with its current strategy $\left(I_{C}<\right.$ threshold $\left.T A\right)$, it will consider an alternative one. If the agent is dissatisfied long enough (its dissatisfaction duration since being stable $D D>T D$ ) and its satisfaction evaluation of the alternative is better enough than that with its current one $\left(I_{A}>I_{C}(1+\right.$ threshold $\left.T O)\right)$, it will change strategy. As stated in equation (1), satisfaction $I$ with a strategy is the average sum of the related attitude $A$ and subjective norm SN.

In equation (1), attitude $\left(A_{S}\right)$ represents an agent's personal view of the difference between its experience and the (potential) outcome of evaluated strategy $S$. The agent $f$ 's experience is its average outcome on the farm $\left(\overline{T_{C, 0}}, \overline{T_{C, 1}}\right)$ with its current farming strategy $\left(S^{f}\right)$ in memory $(M)$. It is computed like this:

$$
\overline{T_{C, l}}=\frac{\sum_{t \text { and } S^{t}=S^{f}}^{M} T_{i}^{t}}{N b\left(S^{t}=S^{f}\right)}
$$

The evaluated outcome depends on the strategy to be evaluated. For agent $f$ 's current farming strategy evaluation, the evaluated outcome is $f$ 's current outcome $\left(T_{i}^{f}\right)$.

Agent $f$ 's attitude toward the current farming strategy $\left(A_{C}\right)$ is like this:

$$
A_{C}^{f}=\sum_{i=1}^{2}\left(W_{i}^{f}\left(T_{i}^{f}-\overline{T_{C, l}^{f}}\right)\right)
$$

If an agent's outcome on its farm changes and this change is considered better than its experience, $A_{C}$ will be positive and strengthen the agent's decision to keep its current strategy. Otherwise, $A_{C}$ will be negative and may influence $I_{C}$. Then the agent may be dissatisfied and evaluate $I_{A}$.

For agent $f$ 's evaluation of an alternative farming strategy, the evaluated outcome is the average outcome $\left(\overline{T_{A, 0}^{f}}, \overline{T_{A, 1}^{f}}\right)$ of other agents having similar characteristics as $f$ but applying the alternative. The similarity is defined by a function $Y$ over the farm's characteristics and is compared with a distance threshold (simi). $Y$ is designed in the model's implementation (see 2.2). The evaluated outcome is computed as follows:

$$
\overline{T_{A, l}^{f}}=\frac{\sum_{Y_{v}^{f}<\text { simi and } S^{v} \neq S^{f}}^{N} T_{i}^{v}}{N b\left(Y_{v}^{f}<\operatorname{simi} \text { and } S^{v} \neq S^{f}\right)}
$$

If there is no corresponding peer (no other agents similar to $f$ and applying the alternative strategy), agent $f$ will search the media for a stereotypical farm as the alternative.

$$
\overline{T_{A, l}^{f}}=T_{\text {model }, i}^{f}
$$

Therefore, agent $f$ 's attitude toward an alternative is: 


$$
A_{A}^{f}=\left\{\begin{array}{c}
0 \text { if }\left(I_{C}^{f}>T A\right) \\
\sum_{i=1}^{2}\left(W_{i}^{f}\left(\overline{T_{A, l}^{f}}-\overline{T_{C, l}^{f}}\right)\right) \text { otherwise }
\end{array}\right.
$$

Another component of satisfaction: the subjective norm, represents how an agent considers others' opinions on the evaluated farming strategy through outcomes, i.e. the strategy's implementation results. It is thus an agent's perceived difference between the outcome to be evaluated and the average of other agents' outcomes.

For agent $f$ 's evaluation of current farming strategy, the subjective norm is:

$$
S N_{C}^{f}=\sum_{i=1}^{2}\left(W_{i}^{f}\left(T_{i}^{f}-\frac{\sum_{v \neq f}^{N}\left(C_{v}^{f} T_{i}^{v}\right)}{\sum_{v \neq f}^{N} C_{v}^{f}}\right)\right)
$$

An agent will be socially satisfied if it perceives that other agents, especially those to whom it lends great credibility ("important others"), consider it as a 'good farmer'. The agent may be so satisfied to have a good social image that it will never consider a major change. Otherwise, if the agent feels socially bad, it may try to become more similar to others in the group or to change of group. This can be done with a change of strategy.

For the evaluation of an alternative farming strategy, the subjective norm is:

$$
S N_{A}^{f}=\left\{\begin{array}{c}
0 \text { if }\left(I_{C}^{f}>T A\right) \\
\sum_{i=1}^{2}\left(W_{i}^{f}\left(\overline{T_{A, l}^{f}}-\frac{\sum_{v \neq f}^{N}\left(C_{v}^{f} T_{i}^{v}\right)}{\sum_{v \neq f}^{N} C_{v}^{f}}\right)\right) \text { otherwise }
\end{array}\right.
$$

If in other agents' opinions, especially those to whom agent $f$ lends great credibility ("important others"), the alternative is not better, then it is judged not good enough to improve the situation. Agent $f$ will tend to keep its current strategy. Otherwise, the agent's subjective norm strengthens its intention to change its strategy.

If an agent changes its strategy, it also changes the importance given to each dimension of practice. The parameter $W$ is the conventional farmers' initial importance given to the productivity dimension.

$$
\text { For conventional agents: } W_{0}=W ; W_{l}=1-W
$$

$$
\text { For organic agents: } W_{0}=1-W ; W_{l}=W
$$

Practice update.

As farmers co-construct their practices [26], at each time $t$, a farmer agent updates its practice by copying the performance of its credible peers with a similar farm.

$$
\Delta P_{i}^{f}=\frac{\sum_{v \neq f \text { and } Y_{v}^{f}<\operatorname{simi} i}^{N} C_{v}^{f}\left(P_{i}^{v}-P_{i}^{f}\right)}{\sum_{v \neq f \text { and } Y_{v}^{f}<\operatorname{simi}}^{N} C_{v}^{f}}
$$

Both dimensions of practice are between 0 and 1. A farmer's productivity performance is also limited by the reference on its farm. 


$$
P_{0}^{f, t+1}=\left\{\begin{array}{c}
0 \text { if }\left(P_{0}^{f, t}+\Delta P_{0}^{f}<0\right) \\
R^{f} \text { if }\left(\left(P_{0}^{f, t}+\Delta P_{0}^{f}\right)>R^{f}\right) \\
P_{0}^{f, t}+\Delta P_{0}^{f} \text { otherwise }
\end{array}\right.
$$

A special case: if agent $f$ looks for an alternative and cannot find a similar peer applying an alternative strategy, it will look for an alternative in the media. If after evaluation, agent $f$ adopts the alternative found in the media, it will also copy the practice.

\subsection{The design of use cases based on agricultural data}

The model is implemented on data from Agricultural General French Census (RGA 2000) about six French "cantons" with strong variations in terms of practice intensity and homogeneity: 1516, Ruynes-en Margeride; 4202, Boën; 4224, Saint-GenestMalifaux; 2208, Chèze; 2235, Plouagat; 2522, Quingey. The initialization of population distributions in each "canton" is deterministic. For sake of simplicity and according to expertise and literature [23], [24], a farmer's farm and practice are defined by three variables: the utilized agricultural area $(U A A)$, the number of dairy cows $(N C)$, and the quota $(Q)$ which is a synthetic indicator of the farm's maximum milk volume. Figure 3 shows the average value of these variables in each "canton".
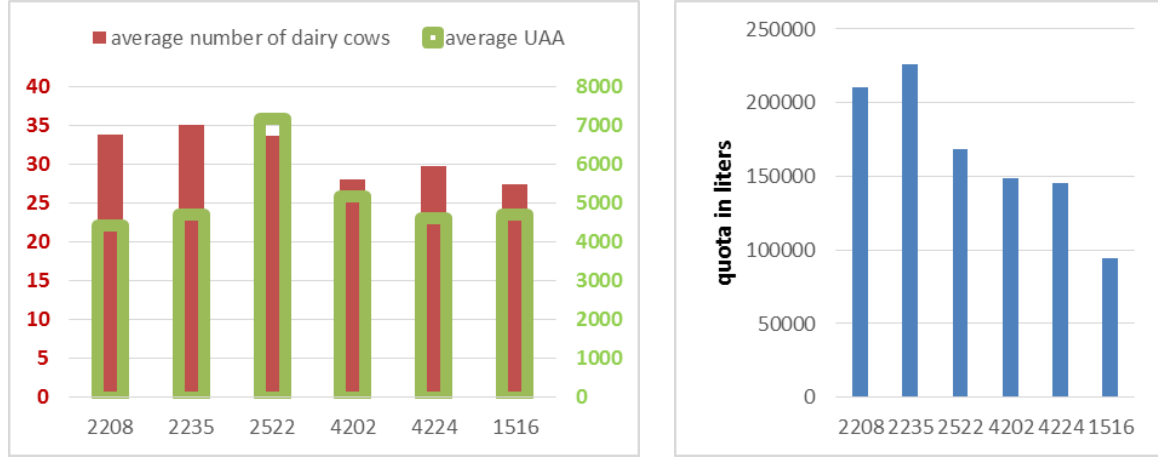

Fig. 3. Average number of dairy cows $N C$, average $U A A$ (in are) and quota (in liters) for the six chosen French "cantons".

The extensity (versus intensity) is here measured as the average $U A A$ /average $N C$. "Cantons" 2208 and 2235 are the biggest producers and most intensive, while 4202, 4224 and 1516 have the least milk production. Despite its average milk production, 2522 is the most extensive "canton", also having the largest average UAA.

The implementations of a farmer, a farm and the media are presented as follows.

Farmer.

A farmer agent is designed by its practice with productivity performance $P_{0}$ and environmental performance $P_{1}$ designed from data. $P_{0}$ is directly deduced from the farm's initial characteristics and corresponds to the normalized average milk volume pro- 
duced by one cow in one year. For farmer/farm $f$, at the initial time $t=0, P_{0}^{f}=Q^{f} / N C^{f}$. At every time $t, P_{i}^{f}=T_{i}^{f} / S A U^{f}$, the equation (15) is only used to update $P_{0}^{f}$.

The $Y$ function telling how two farmers are judged similar is based on a similarity of their farms' characteristics regarding $U A A$ and $N C$. For agent $f$, agent $v$ is a similar peer if $\frac{\left|U A A^{f}-U A A^{v}\right|}{U A A^{f}}<\operatorname{simi}$ and $\frac{\left|N C^{f}-N C^{v}\right|}{N C^{f}}<\operatorname{simi}$. The threshold simi is a parameter and supposed as 0.1 in the model.

Farm.

As shown above, each farm is initialized by the crossed distribution of discretized utilized agricultural areas $(U A A)$ and quotas $(Q)$ of its "canton" from the RGA 2000. They remain constant all along the simulation. The number of dairy cows $(N C)$ is computed from a law extracted from data (with a regression $\mathrm{r}^{2}=0.9563$ ):

$$
N C=0.2463 U A A+0.0001106 Q
$$

From databases regarding farmers' production and various sources ${ }^{1}$, a law is built to compute the potential maximum milk production of an organic farm starting with the conventional strategy, knowing its initial productivity performance $P_{0}$ after the normalization and $P_{0}$ ' before the normalization. A farm $f$ 's normalized reference $R$ and reference $R$ ' before the normalization are computed as follows:

For a conventional farm: $R=P_{0}(t=0)$

For an organic farm: $R^{\prime}=0.6046 P_{0}{ }^{\prime}(t=0)+1913 N C$

The environmental amenities outcome $T_{1}$ is computed at every time by an aggregated function of literature [23], [24]. It considers mineral impacts and energy consumption related to the total milk production and the farm's agricultural surface:

For a conventional farm: $T_{1}=\left(53 U A A+2.918 T_{0}\right) / 2$

For an organic farm: $T_{1}=\left(-10 U A A+2.588 T_{0}\right) / 2$

Using French dairy farms' database in RGA $2000, R$ ' is to be normalized between 0 (very low production) and 1 (very high production). $53 U A A, 2.918 T_{0},-10 U A A$ and $2.588 T_{0}$ are normalized values between 0 and 1 . The normalization is:

$$
x=\left(x^{\prime}-\min \right) /(\max -\min )
$$

With: $\min =$ minimum real value in the database; $\max =$ maximum real value in the database; $x$ is the normalized value of real value $x^{\prime}$.

\section{The media.}

We use laws extracted from data to design farmers' alternative models. When a conventional farmer $f$ wants to evaluate the organic strategy at time $t+1$, it computes $T_{0}{ }^{\prime}(t+1)$ as follows and its related $T_{1}{ }^{\prime}(t+1)$ with the equation (20). Noting that $T_{0}{ }^{\prime}$ is the farmer's real productivity outcome on the farm (before the normalization).

\footnotetext{
${ }^{1}$ http://www.cantal.chambagri.fr/fileadmin/documents/Internet/Autres\%20articles/pdf/2014/Bio /ABBL2008-2012.pdf

http://www.tech-n-bio.com/

http://www.agrobio-bretagne.org/
} 


$$
T_{0}{ }^{\prime}(t+1)=0.6046 T_{0}{ }^{\prime}(t)+1913 N C
$$

When an organic farmer $f$ wants to evaluate the conventional strategy at time $t+1$, it computes $T_{0}{ }^{\prime}(t+1)$ as follows and its related $T_{I}{ }^{\prime}(t+1)$ with the equation (21).

$$
T_{0}{ }^{\prime}(t+1)=\left(T_{0}{ }^{\prime}(t)-1913 N C\right) / 0.6046
$$

\subsection{Experimental design}

[15] has studied 625 parameter sets identifying all the qualitative behaviors of the model to diagnose reasons for the absence of conversion. This work aims to study conversion in six different French "cantons" using the same 625 parameter sets varying the main parameters: $\alpha$ (slope of logistic function) from 5 to $41, T A$ (threshold to consider an alternative) from 0.41 to $0.49, T O$ (threshold to consider an alternative) from 0.01 to $0.09, W$ (importance given to the dimension representing farming strategy) from 0.6 to 1 . $T D$ (threshold for two counters of duration) is kept constant with the value 5 . Agents' memory is also kept constant as 10 years. The distance for similarity, simi, values 0.1 . The evolution of each "canton" with a population of 100 conventional farmers (no organic farmers at first) is simulated for 30 years and replicate 100 times.

\section{Model behaviors}

The adoption situations in each "canton" are studied as follows.

\subsection{The adoption rate and "cantons"}

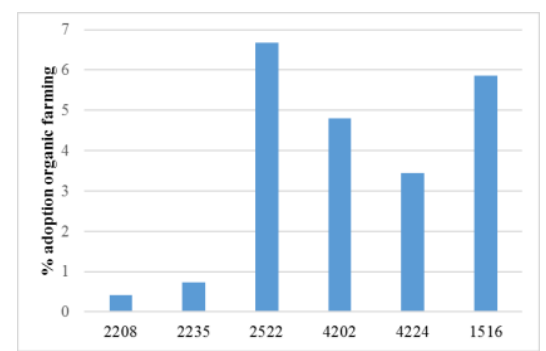

Fig. 4. - Average percentage of organic farming adoption according to "canton" over 625 sets of parameters and their related 100 replications

At the initialization, each "canton" has no organic farm. Figure 4 shows that farms in different "cantons" have different adoption percentages after 30 years. "Canton" 2522 convert the most, followed by 4202 and 1516, and then 4224. 2208 and 2235 convert the least. As these six "cantons" have different farm characteristics. The population distribution is studied to learn more about the conversion rate. 


\subsection{The characteristics of "cantons"}

As shown in "Materials and methods" section, each farm has a couple of outcomes (total production) on two dimensions: productivity outcome $\left(T_{0}\right)$ and environmental outcome $\left(T_{1}\right)$. These two values indicate directly a farm's utilized agricultural area $(U A A)$ and quota $(Q)$ which are constant characteristics along the simulation. With another constant characteristic on the farm: number of cows $(N C)$, a farmer's initial practice performance: productivity performance $\left(P_{0}\right)$ and environmental performance $\left(P_{1}\right)$ are also defined. In figure 5, for each canton, its average initial $T_{0}$ and $T_{1}$ are shown on the left; its average initial $P_{0}$ and $P_{l}$ are shown on the right. The black lines in each histogram show the variation.

Consistently with figure 3 , figure 4 and 5 show that "cantons" adopting the least: 2208 and 2235, are the most intensive and productive (having the smallest $U A A / N C$ and the largest initial $T_{0}$ ). They are followed by 4224, 4202 and 1516, which have the largest initial environmental outcome $T_{1}$ and lower productivity outcome $T_{0}$. The "canton" with the most adoption: 2522, is the most extensive (figure 3), but having the worst initial environmental performance $P_{l}$ (figure 5). Its outcomes: $T_{0}$ and $T_{1}$ are medium.

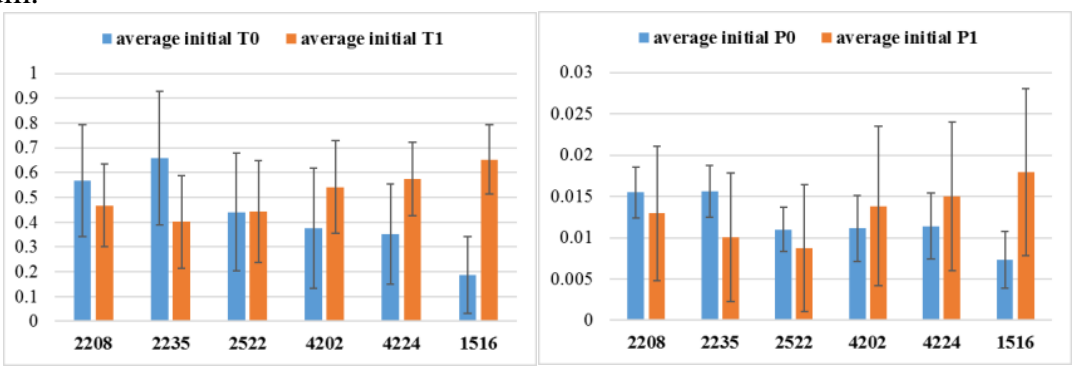

Fig. 5. - Average of initial $T_{0}$ and $T_{l}$ (left panel) and $P_{0}$ and $P_{l}$ (right panel) in each "canton". Error bars indicates "more or less" one standard deviation.

The credibility a farmer gives to another depends on their relative outcomes $\left(T_{0}\right.$ and $T_{1}$ ). The social evaluation of satisfactions depends also on their relative $T_{0}, T_{1}$ and on the outcomes of its "similar" farmers (equation 11 and 12). Table 1 shows the initial farm distributions of each "canton" in terms of $T_{0}$ and $T_{1}$.

Table 1. Initial farm distributions according to "cantons" and farm types in terms of $T_{0}$ or $T_{l}$

\begin{tabular}{|c|c|c|c|c|c|c|c|c|c|c|c|c|c|}
\hline initlal 70 santon & 2208 & 2235 & 2522 & 4202 & 4224 & 1516 & initial F1 canton & 2208 & 2235 & 2522 & 4202 & 4224 & 1516 \\
\hline 0.05 & 3 & 3 & 5 & 16 & 17 & 37 & 0.85 & 3 & 3 & 3 & 13 & 14 & 19 \\
\hline 0.15 & 3 & 2 & 12 & 6 & 5 & 27 & 0.75 & 5 & 5 & 7 & 5 & 7 & 29 \\
\hline 0.25 & 4 & 7 & 14 & 22 & 21 & 17 & 0.65 & 14 & 5 & 15 & 22 & 21 & 21 \\
\hline 0.35 & $\frac{8}{25}$ & $\frac{2}{21}$ & $\frac{16}{15}$ & $\frac{14}{18}$ & $\frac{18}{12}$ & $\frac{9}{5}$ & 0.55 & 25 & 22 & 16 & 19 & 26 & 14 \\
\hline$\frac{0.45}{0.55}$ & $\frac{25}{18}$ & $\frac{21}{12}$ & $\frac{15}{16}$ & $\frac{18}{9}$ & $\frac{12}{14}$ & $\frac{5}{2}$ & 0.45 & 24 & 19 & 23 & 24 & 23 & 10 \\
\hline 0.65 & 11 & 6 & 10 & 3 & 10 & 2 & 0.35 & 15 & 7 & 10 & 4 & 6 & 5 \\
\hline 0.75 & 9 & 3 & $\frac{5}{2}$ & 2 & $\frac{\pi}{2}$ & 1 & 0.25 & 5 & 23 & 11 & 6 & 1 & 2 \\
\hline 0.85 & 8 & 10 & 2 & 6 & 1 & 0 & 0.15 & 7 & 14 & 5 & 4 & 2 & 0 \\
\hline 0.95 & 11 & 34 & 8 & 4 & 0 & 0 & 0.05 & 2 & 2 & 10 & 3 & 0 & 0 \\
\hline
\end{tabular}


"Cantons" with the most converted agents: 2522, has medium average $T_{0}$ and $T_{1}$ while its distribution is diverse. The other "cantons" have more concentrated distributions. "Cantons" 2208 and 2235 have few farms with low $T_{0}$. "Cantons" 4224, 4202 and 1516 have numerous farms with high $T_{1}$ and very few farms with low ones.

\subsection{The adoption rate and farm types}

The adoption rate varies with "cantons". In fact, it varies also with initial outcome types in terms of productivity outcome $\left(T_{0}\right)$ and environmental outcome $\left(T_{1}\right)$.

Generally, the probability to convert is high for cases with numerous farms at the initialization. Farms with a low initial $T_{0}$ or a high initial $T_{1}$ (first lines in Table 2) convert more than others. However, for the highest initial $T_{1}$, the conversion rate is not the largest (except in 2208 and 2235).

The distributions of adoption vary with "cantons". 2522 has a disperse distribution and its adoptions center on medium outcomes. 4202, also having a disperse distribution, has adoptions in cases with high $T_{1}$ and low $T_{0} .1516$ and 4224 have a centered distribution and their adoptions are rather in cases with very low $T_{0}$ and high $T_{l}$.

Table 2. Average percentage of organic farming adoption according to "canton" and farm types in terms of $T_{0}$ or $T_{1}$ over 625 sets of parameters and their related 100 replications

\begin{tabular}{|c|c|c|c|c|c|c|c|c|c|c|c|c|c|c|}
\hline initial FO canton & 2208 & 2235 & 2522 & 4202 & 4224 & 1516 & initial T1 canton & 2208 & 2235 & 2522 & 4202 & 4224 & 1516 \\
\hline 0.05 & 0.29 & 0.36 & 0.93 & 2.37 & 2.26 & 4.19 & 0.85 & 0.29 & 0.36 & 0.25 & 1.41 & 1.39 & 1.16 \\
\hline 0.15 & 0.06 & 0.07 & 1.91 & 0.68 & 1.05 & 1.98 & 0.75 & 0.08 & 0.15 & 0.93 & 1.07 & 1.07 & 2.69 \\
\hline 0.25 & 0.02 & 0.26 & 1.91 & 1.75 & 0.65 & 1.28 & 0.65 & 0.08 & 0.02 & 2.12 & 1.34 & 0.46 & 2.27 \\
\hline 0.35 & 0.02 & 0.01 & 2.02 & 0.78 & 0.32 & 0.55 & 0.55 & 0.02 & 0.27 & 2.07 & 1.63 & 1.48 & 1.18 \\
\hline 0.45 & 0.06 & 0.17 & 1.04 & 0.72 & 0.17 & 0.25 & 0.45 & 0.01 & 0.17 & 1.87 & 0.98 & 0.26 & 0.96 \\
\hline 0.55 & 0.11 & 0.07 & 1.4 & 0.33 & 0.16 & 0.15 & 0.45 & 0.35 & 0.11 & 0.01 & 1 & 0.15 & 0.07 & 0.28 \\
\hline 0.65 & 0.02 & 0.01 & 0.59 & 0.06 & 0.12 & 0.16 & 0.25 & 0 & 0.02 & 1.31 & 0.23 & 0.03 & 0.05 \\
\hline 0.75 & 0 & 0 & 0.1 & 0.05 & 0 & 0.02 & 0.25 & 0.15 & 0.01 & 0.01 & 0.37 & 0.05 & 0 & 0 \\
\hline 0.85 & 0 & 0.01 & 0.06 & 0.1 & 0.03 & 0 & 0.05 & 0.05 & 0 \\
\hline 0.95 & 0.01 & 0.04 & 0.23 & 0.01 & 0 & 0 & 0.05 & 0 & 0.01 & 0.29 & 0 & 0 & 0 \\
\hline
\end{tabular}

"Cantons" converting the least, exhibit distribution profiles with one higher density for medium $T_{0}$ and another one for the highest $T_{0}$. They are rather homogeneous in $T_{1}$.

\section{Conclusion discussion}

[14] and [15] argue that the organic farming adoption is strongly influenced by the imitation of "important others" practices and is very sensitive to the distance between farms. However, the model proposed and studied in [15] considers only prototypical populations and points out the reasons for non-adoption of organic farming. In this paper, we focus on the impact of population characteristics on the adoption degree. In this work, the model is explored further by an initialization with six French "cantons" (about 100 dairy farmers) varying in their farm type distributions from the French RGA 2000.

At the farm level, we have observed that farms with low milk total production and high environmental total production convert more than others. Such farms are said having extensive practices and being more susceptible to convert according to ex- 
perts. Approximately one-third of global land under organic production is located in unfavorable areas where smallholder farmers, who often lack access to insurance or inexpensive credit, dominate agricultural production [25]. Keeping their extensive practices, or even increasing this extensity, by adopting for example organic farming, can be beneficial to eliminate their reliance on expensive, fossil fuel-derived chemical inputs [26] [27]. This increasing autonomy is taken into account in our environmental outcome which considers the decreasing of inputs and energy consumption.

At the regional level, intensive regions convert less than extensive ones, especially those with not very good environmental outcomes. Indeed, we notice that the most intensive "cantons" almost do not convert (those located in the Brittany region). They are more likely to be satisfied with their current situation and do not consider a major change. Despite of having the least productivity outcomes, "cantons" 1516, 4202 and 4224 do not have the most conversion because of their already good environmental outcome. The adoption of organic farming cannot bring them much environmental gain. On the contrary, the most extensive "canton", 2522 has the largest adoption rate. It is less productive than farms in the Brittany region (2238 and 2235), but more than other "cantons". Its environmental production is not as good as the other extensive "cantons" (1516, 4202 and 4224). Thus, most farms from this "canton" can poorly decrease their productivity outcome and increase much their environmental outcome with the conversion to organic farming. This result can sound strange but it has been pointed out by [34] that most farmers having already chosen low-input strategy (i.e. strongly extensive) do not convert since they cannot improve more their environmental impact. The only reason they can convert is for economic purpose in case of strong crisis. We also show that more diverse regions, having high densities for different farm types, tend to adopt more but this point deserves more investigations. In fact, our model can also be applied to other contexts, for example, other forms of adoption in agriculture.

However, we cannot compare our adoption rates for the chosen French regions: firstly, because the data are not so easy to obtain; secondly very important dynamics are not present in the model and make the comparison having no sense. Adding to the model external factors such as economic crisis, but also demographic evolution implying an increasing average farm sizes, are the next steps for research about this model.

\section{$5 \quad$ Reference}

[1] M.-S. Dedieu, A. Lorge, O. Louveau, and V. Marcus, "Les exploitations en agriculture biologique: quelles performances économiques?," 2017.

[2] J. Sainte-Beuve, D. Bougherara, and L. Latruffe, "Performance économique des exploitations biologiques et conventionnelles: Levier économique à la conversion," 10p Transversalités L'agriculture Biol. Strasbg., 2011.

[3] L.-A. Sutherland, R. J. F. Burton, J. Ingram, K. Blackstock, B. Slee, and N. Gotts, "Triggering change: Towards a conceptualisation of major change processes in farm decision-making," J. Environ. Manage., vol. 104, pp. 142-151, août 2012.

[4] L. Rickards and S. M. Howden, "Transformational adaptation: agriculture and climate change," Crop Pasture Sci., vol. 63, no. 3, p. 240, 2012. 
[5] A.-M. Dowd, N. Marshall, A. Fleming, E. Jakku, E. Gaillard, and M. Howden, "The role of networks in transforming Australian agriculture," Nat. Clim. Change, vol. 4, no. 7, pp. 558-563, Jun. 2014.

[6] J. R. Fairweather, L. M. Hunt, C. J. Rosin, and H. R. Campbell, “Are conventional farmers conventional? Analysis of the environmental orientations of conventional New Zealand farmers," Rural Sociol., vol. 74, no. 3, pp. 430-454, 2009.

[7] I. Darnhofer, W. Schneeberger, and B. Freyer, "Converting or not converting to organic farming in Austria:Farmer types and their rationale," Agric. Hum. Values, vol. 22, no. 1, pp. 39-52, Mar. 2005.

[8] D. Rigby, T. Young, and M. Burton, "The Development of and Prospects for Organic Farming in the UK," Food Policy, vol. 26, pp. 599-613, Dec. 2001.

[9] J. Pavie, A.-C. Dockès, and L. Echevarria, Etude des freins à la conversion à l'agriculture biologique des exploitations laitières bovines. Paris Cedex 12: Institut de l'Elevage, 2002.

[10] C. Barbier, M. Cerf, and J.-M. Lusson, "Cours de vie d'agriculteurs allant vers l'économie en intrants: les plaisirs associés aux changements de pratiques," Activités, vol. 12, no. 12-2, 2015.

[11] M. Burton, D. Rigby, and T. Young, "Modelling the adoption of organic horticultural technology in the UK using duration analysis," Aust. J. Agric. Resour. Econ., vol. 47, no. 1, pp. 29-54, 2003.

[12] R. L. Goldstone and M. A. Janssen, "Computational models of collective behavior," Trends Cogn. Sci., vol. 9, no. 9, pp. 424-430, Sep. 2005.

[13] V. Grimm, "Ten years of individual-based modelling in ecology: what have we learned and what could we learn in the future?," Ecol. Model., vol. 115, no. 2, pp. 129-148, Feb. 1999.

[14] S. Huet, C. Rigolot, Q. Xu, Y. De Cacqueray-Valmenier, and I. Boisdon, "Toward Modelling of Transformational Change Processes in Farm Decision-Making," Agric. Sci., vol. 09, no. 03, pp. 340-350, 2018.

[15] Q. Xu, S. Huet, C. Poix, I. Boisdon, and G. Deffuant, "Why do farmers not convert to organic farming? Modelling conversion to organic farming as a major change," Nat. Resour. Model., 2018.

[16] P. Kaufmann, S. Stagl, and D. W. Franks, "Simulating the diffusion of organic farming practices in two New EU Member States," Ecol. Econ., vol. 68, no. 10, pp. 2580-2593, août 2009.

[17] I. Ajzen and M. Fishbein, Belief, attitude, intention and behavior: An introduction to theory and research. Reading, MA: Addison-Wesley, 1975.

[18] H. Mintzberg, D. Raisinghani, and A. Theoret, "The Structure of 'Unstructured' Decision Processes," Adm. Sci. Q., vol. 21, no. 2, p. 246, juin 1976.

[19] B. Öhlmér, K. Olson, and B. Brehmer, "Understanding farmers' decision making processes and improving managerial assistance," Agric. Econ., vol. 18, no. 3, pp. 273-290, 1998.

[20] J.-P. Darré, La parole et la technique: l'univers de pensée des éleveurs du Ternois, vol. 10. Editions L'Harmattan, 1985.

[21] V. Grimm et al., "ODD protocol: A standard protocol for describing individual-based and agent-based models," Ecol. Model., vol. 198, no. 1-2, pp. 115-126, Sep. 2006.

[22] E. M. Rogers, Diffusion of innovations, 3rd ed. New York: London: Free Press ; Collier Macmillan, 1983.

[23] J. Pavie, H. Chambaut, E. Moussel, J. Leroyer, and V. Simonin, "Evaluations et comparaisons des performances environnementales, économiques et sociales des systèmes bovins biologiques et conventionnels dans le cadre du projet CedABio," Renc Rech Rumin., vol. 19, pp. 37-40, 2012. 
[24] H. Chambaut et al., "Profils environnementaux des exploitations d'élevage bovins lait et viande en agriculture biologique et conventionnelle: enseignements du projet CedABio," Rencontres Autour Rech. Sur Rumin., pp. 53-56, 2011.

[25] F. IFOAM, "The World of Organic Agriculture: Statistics \& Emerging Trends 2017," 2017.

[26] D. Pimentel, P. Hepperly, J. Hanson, D. Douds, and R. Seidel, "Environmental, Energetic, and Economic Comparisons of Organic and Conventional Farming Systems," BioScience, vol. 55, no. 7, p. 573, 2005.

[27] H. Uematsu and A. K. Mishra, "Organic farmers or conventional farmers: Where's the money?," Ecol. Econ., vol. 78, pp. 55-62, Jun. 2012.

[28] H. Eakin, "Smallholder maize production and climatic risk: A case study from Mexico," Clim. Change, vol. 45, no. 1, pp. 19-36, 2000. 